\title{
Refractory Anemia with an Excess of Blasts Developed into Overt Leukemia with Leukothrombocytosis
}

\author{
Isao TaKahashi, Kazuto Togitani, Motoharu Yokoyama, Tetsunori Aita, Kiyoshi Makihata, \\ Kazutaka Sunami, Masayoshi Shiromoto, Toru Nakamura, Ichiro Kawada, Masaaki Nishimura, \\ Hiroshi NaKada, Seiichi Yorimitsu, Susumu Miyake, Ken-ichi Machida and Ichiro Kubonishi*
}

A 72-year-old man with refractory anemia with an excess of blasts developed overt leukemia with leukothrombocytosis. Hematological and physical findings closely resembled those of an accelerated or blastic phase of chronic myelocytic leukemia. The cytogenetic anomaly of $i(17 q)$ was observed during the course. The present case is suggestive of the diversities of myelodysplastic syndromes (MDS), including relationships between MDS and myeloproliferative disorders (MPD) and acute leukemia.

(Internal Medicine 34: 28-31, 1995)

Key words: myelodysplastic syndromes, myeloproliferative disorders, isochromosome 17q

\section{Introduction}

Myelodysplastic syndromes (MDS) and myeloproliferative disorders (MPD) are thought to be the clonal origin in pluripotent stem cell (1-4). MDS are characterized by cytopenia in peripheral blood, normo- or hyperplastic marrow and morphological anomalies of hematopoietic cells (5). On the other hand, the hematological characteristics of myeloproliferative disorders (MPD) include the increase of some of blood cells in the peripheral blood resulting in leukocytosis, polycythemia or thrombocytosis (6). Thus, one of the main biological differences between MDS and MPD may be whether the maturation disturbance is present or not. In this paper, a case of refractory anemia with an excess of blasts (RAEB) developed overt leukemia with MPD-like findings such as leukocytosis, thrombocytosis and hepatosplenomegaly.

\section{Case Report}

The patient, a 72-year-old man, developed generalized weakness in mid April 1992. He visited another hospital because of weakness; iron was given intravenously under the diagnosis of anemia. On June 9, 1992, he was referred to our hospital because of an unfavorable course. The patient suffered from herpes zoster in 1990. Physical examination on admission revealed a modrately nourished male. Body temperature was $36.5^{\circ} \mathrm{C}$. Pulse was $89 / \mathrm{min}$ regular. Conjunctiva was anemic but not icteric. The heart was normal. Fine crackles were present at the bases of the lungs posteriorly. Hepatosplenomegaly, superficial lymphnodes and hemorrhagic tendency were not observed. The neurological examination was negative. Examination of the blood showed a red blood cell (RBC) count of $183 \times 10^{4} / \mu 1$, hemoglobin $(\mathrm{Hb})$ of $8.6 \mathrm{~g} / \mathrm{dl}$, a platelet $(\mathrm{Plt})$ count of $2.8 \times 10^{4} / \mu 1$, a white blood cell (WBC) count of $5,600 / \mu 1$ with $1 \%$ myeloblasts, $70 \%$ neutrophils, $8 \%$ eosinophils, $2 \%$ monocytes and $9 \%$ lymphocytes. A few erythroblasts and megakaryocytes were found. Giant thrombocytes, neutrophils with hypersegmentation or pseudo-Pelger anomaly were also found (Fig. 1a, b, c). Findings of biopsied specimen of marrow were hyperplastic with an increase in megakaryocytes (Fig. 2a). Bone marrow smear revealed $15.0 \%$ myeloblasts, $6.0 \%$ promyelocytes, $8.8 \%$ myelocytes, $4.4 \%$ metamyelocytes, $28.8 \%$ neutrophils, $8.6 \%$ lymphocytes and $13.2 \%$ erythroblasts. The megakaryocyte count was increased to $540 / \mu \mathrm{l}$. Multinucleated erythroblasts, neutrophils with pseudo-Pelger anomaly and micromegakaryocytes were observed (Fig. 2b,c, d). Cytogenetic analysis was performed by Giemsa-banding technique. Twenty metaphases were analyzed, among which 15 showed a 46,XY, i(17q) karyotype (Fig. 3). Serum levels of asparate aminotransferase (AST), alanine aminotransferase (ALT), alkaline

From the Department of Internal Medicine, Kochi Municipal Central Hospital, Kochi and *the Third Department of Internal Medicine, Kochi Medical School, Kochi

Received for publication April 28, 1994; Accepted for publication September 9, 1994

Reprint requests should be addressed to Dr. Isao Takahashi, the Department of Internal Medicine, Kochi Municipal Central Hospital 2-7-33, Sakuraicho, Kochi 780 


\section{RAEB Developing Leukothrombocytosis}

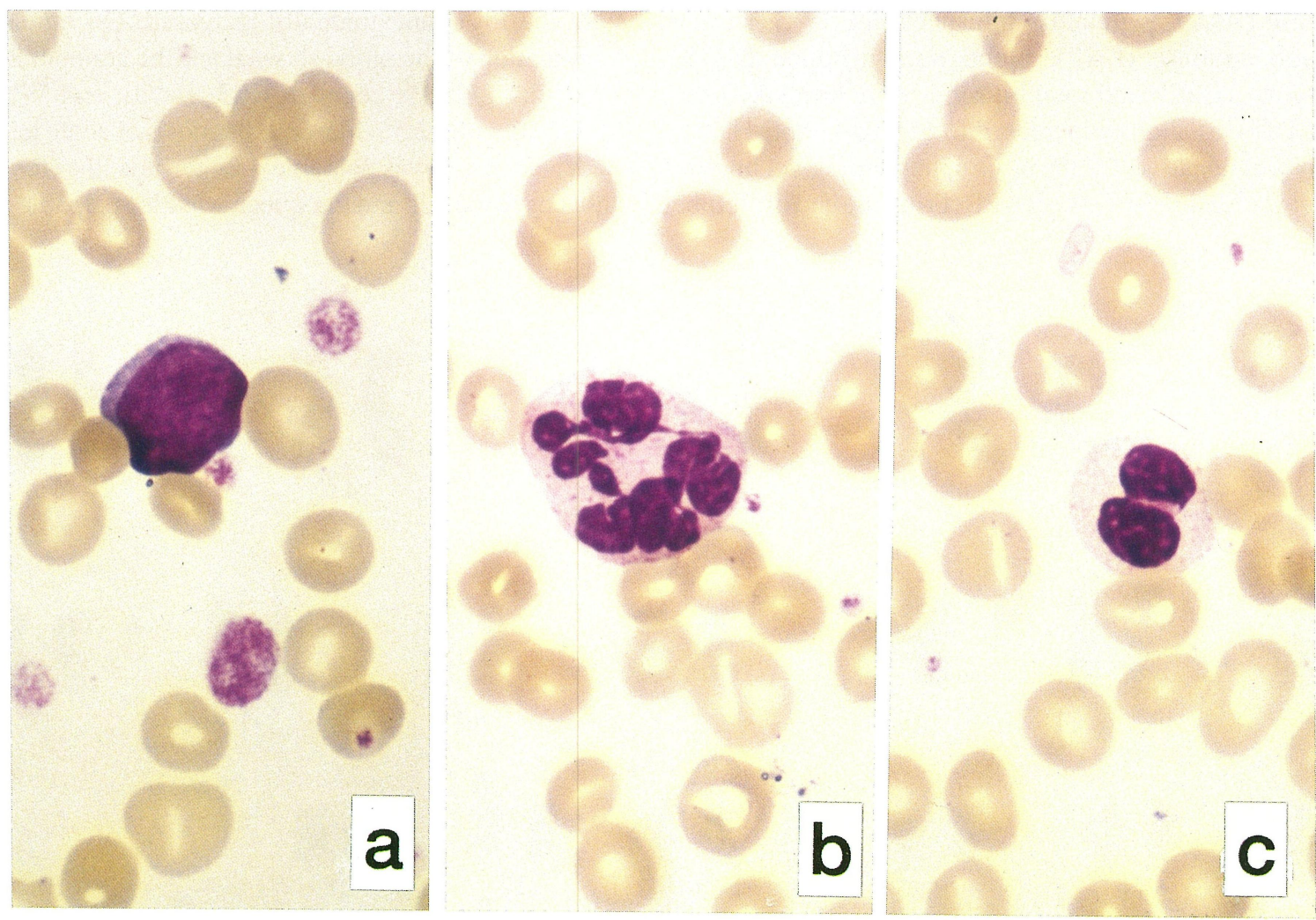

Fig. 1. May-Giemsa staining of peripheral blood cells showing myeloblast and giant thrombocyte (a), hypersegmented neutrophil (b) and neutrophil with pseudo-Pelger anomaly $(\times 330)$.
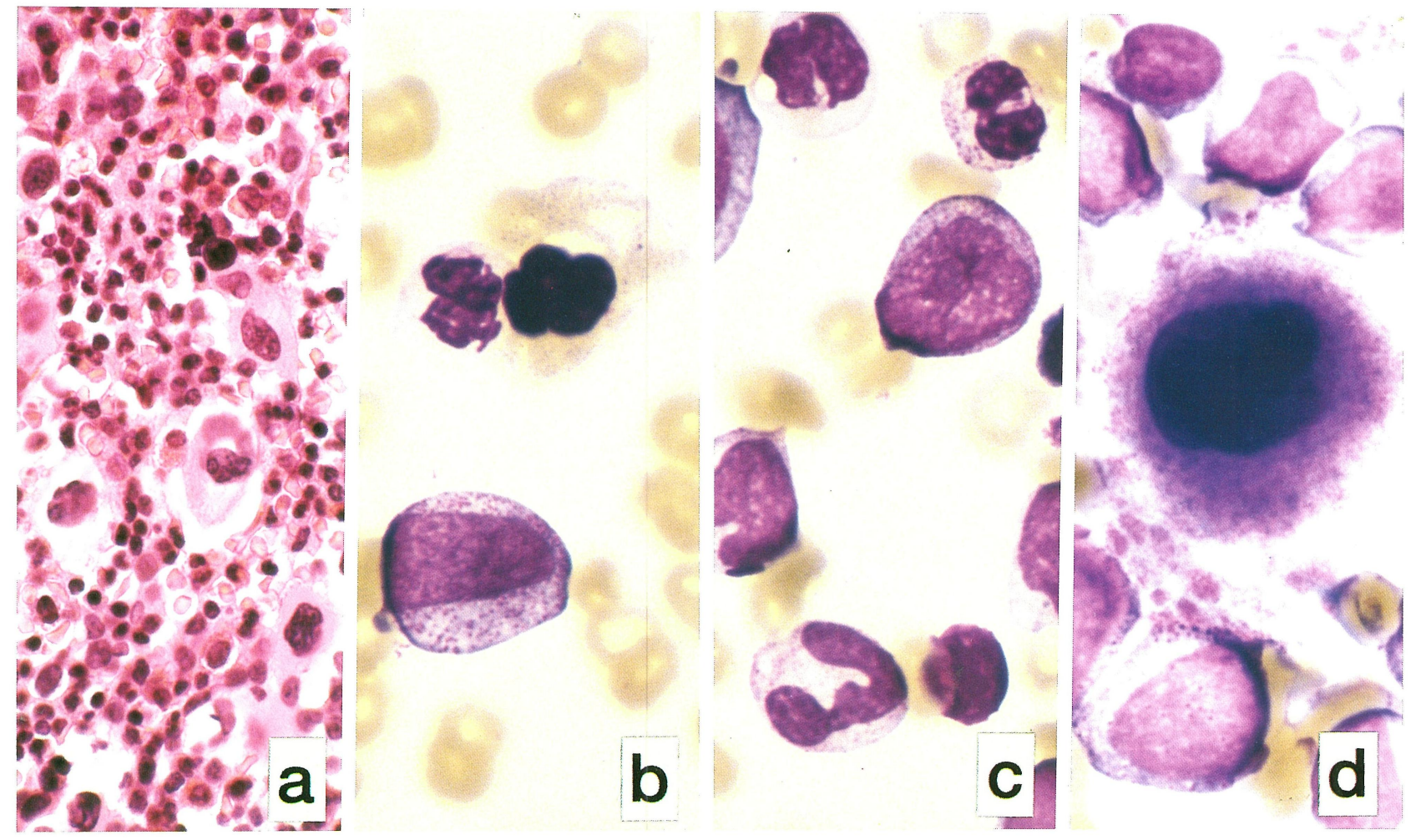

Fig. 2. Biopsied specimen of marrow showing hyperplastic marrow with an increase in megakaryocytes (a) $(\times 132)$. May-Giemsa staining of marrow cells showing a multinucleated erythroblast (b), neutrophils with pseudo-Pelger anomaly (c) and micromegakaryocyte $(d)(\times 330)$. 
phosphatase (ALP), $\gamma$-glutamyl transpeptidase $(\gamma$-GTP), leucine aminopeptidase (LAP), blood urea nitrogen (BUN), cholinesterase $(\mathrm{ChE})$ and total bilirubin were normal. Serum level of LDH was slightly increased to 655 IU/l. Anti-HTLVI antibody was negative. On the basis of hematological findings the patient was diagnosed to be RAEB. From the end of October
1992 the Plt count gradually increased. On January 7, 1993 examination of the blood showed an Rbc count of $216 \times 10^{4} / \mu \mathrm{l}$, $\mathrm{Hb}$ of $6.4 \mathrm{~g} / \mathrm{dl}$, Plt count of $61.3 \times 10^{4} / \mu \mathrm{l}$, and $\mathrm{Wbc}$ count of $42,300 / \mu 1$ with $32.5 \%$ blasts; a diagnosis of acute leukemia was made. The liver was palpable 4 fingerbreadths (fbs) below the right costal margin and the spleen was also palpable $3 \mathrm{fbs}$ below

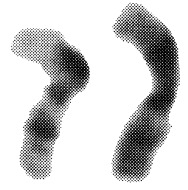

1

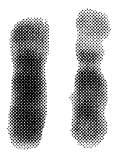

6

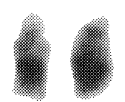

13

19

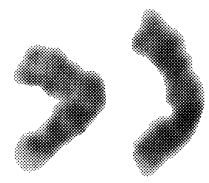

2

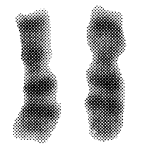

7

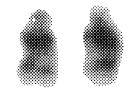

14

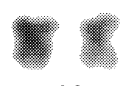

20

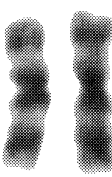

3

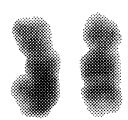

8

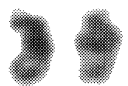

15

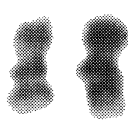

9

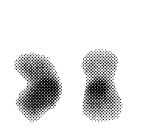

16

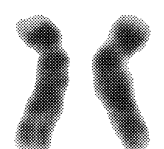

4

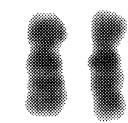

10

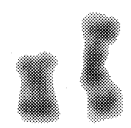

$17 \wedge$

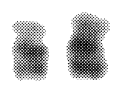

18

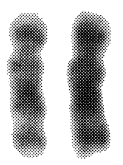

5

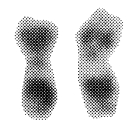

11

12

Fig. 3. Cytogenetic analysis performed by Giemsa-banding technique. Twenty metaphases were analyzed, among which 15 showed a 46, XY, i(17q) karyotype.

\section{H.O. 72 y/o Male MDS (RAEB $\rightarrow$ RAEB-t) $\rightarrow$ AML}

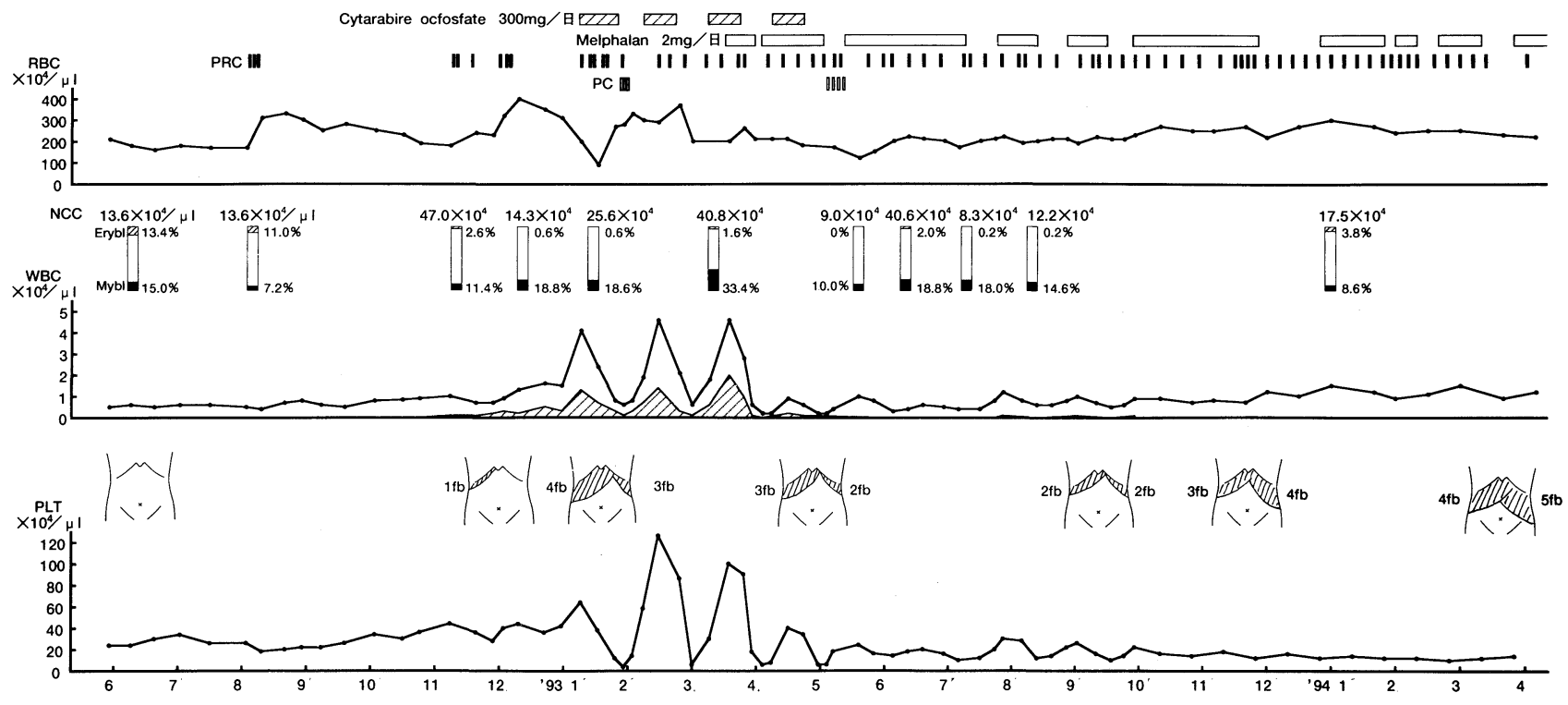

Fig. 4. Hematological course. PRC: Packed red blood cells, PC: Platelet concentrate. 
the left costal margin. The histological examination of bone marrow clot examined on January 11 showed hyperplastic marrow with the increase of blasts and megakaryocytes. From January 12, 1993 the oral administration of $300 \mathrm{mg} /$ day of cytarabine ocfosfate was started. As shown in Fig. 4, Plt count fluctuated with WBC count. On February 7, 1993 the blood examination showed Plt count of $107 \times 10^{4} / \mu \mathrm{l}$ and WBC count of $52,300 / \mu \mathrm{l}$ with $40 \%$ blasts. Results of marker analysis of blasts examined on March 11 were compatible with myeloblasts; CD13 63.9\%, CD33 76.8\% and CD41 0\%. Blasts were also positive for anti-myeloperoxidase monoclonal antibody (AM8) at $60.9 \%$, for AM-9 at $65.2 \%$ and for $\mathrm{AM}-10$ at $70.5 \%$, respectively (7). On March 16, 1993 the oral administration of $2 \mathrm{mg}$ of melphalan was started. Leukothrombocytosis was improved without remission. On March 9, 1994, one year later, blood examination showed an RBC count of $244 \times 10^{4} / \mu \mathrm{l}, \mathrm{Hb}$ of $7.3 \mathrm{~g} / \mathrm{dl}$, Plt count of $18.3 \times 10^{4} / \mu \mathrm{l}$ and WBC count of $20,200 /$ $\mu 1$ with $6.5 \%$ blasts, $5.5 \%$ promyelocytes, $4.0 \%$ myelocytes, $1.5 \%$ metamyelocytes, $16.5 \%$ neutrophils, $2.0 \%$ eosinophils, $11.0 \%$ basophils, $39.0 \%$ monocytes and $14 \%$ lymphocytes. The liver and spleen were palpable $4 \mathrm{fbs}$ and $5 \mathrm{fbs}$, respectively. Sequential analyses of chromosomes showed 47,XY,i(17q) in all of 20 cells examined. The patient is alive and was treated with transfusion and intermittent administration of melphalan.

\section{Discussion}

MDS which are hematological disorders with the potential of progression to acute leukemia, presumably result from the transformation of pluripotent stem cells giving rise to cytopenia, morphological anomalies and functional defects of erythroid, myeloid and megakaryocytic cells. MDS are classified into 5 groups: refractory anemia (RA), RA with ringed sideroblasts (RARS), RAEB, RAEB in transformation (RAEBt) and chronic myelomonocytic leukemia (CMML) (5). MPD is also thought to be a clonal disorder arising from a neoplastic transformation at the level of the pluripotent stem cell, resulting in an increase in some of the blood cells in the peripheral blood $(4,6)$. Thus, one of the differences in biological aspects between MDS and MPD is the presence or lack of a maturation disturbance. Although the appearance of MPD during the course of MDS is rare, some MDS showing MPD-like findings have been reported. Beris et al (8) reported a case developing polycythemia vera and two cases developing thrombocytosis. MDS cases developing leukothrombocytosis have been reported by Economopoulos et al (9), Yamaguchi et al (10) and Ohyashiki et al (11). Additional diagnoses such as Philadelphia chromosome-negative chronic myelocytic leukemia $\left(\mathrm{Ph}^{1-} \mathrm{CML}\right)$, blastic crisis of $\mathrm{Ph}^{1-} \mathrm{CML}$, unclassified MPD (UMPD) have been given with the appearance of MPD-like characteristics, respectively. The present case also showed leukothrombocytosis during the sequential changes from RAEB to RAEB-t and acute leukemia. Hematological and physical findings such as leukocytosis with the increase of blasts, thrombocytosis and hepatosplenomegaly closely resembled to those observed in the accelerated or blastic phase of CML. Recently, structural rearrangements of the long arm of chromosome 3 (3q anomalies) in hematological malignancies have been reported to be associated with megakaryocytic dysplasia, increased numbers of megakaryocytes and thrombocytosis (12). In the present case both megakaryocytic dysplasia and increased numbers of megakaryocytes were observed, however, sequential analyses of chromosomes revealed an isochromosome for the long arm of chromosome 17 [i(17q)], which has been frequently found prior to clinical or other evidences of blastic crisis of CML (13). Whether some biological bases common to MDS and MPD already coexisted in this case or whether some factors other than $3 q$ further disturbed the status of MDS to evolve into overt leukemia with leukothrombocytosis are questionable. Further studies on these cases are thought to bring insight into the evaluation of the diversities of MDS, including the relationships between MDS, MPD and acute leukemia.

\section{References}

1) Prchal JT, Throckmoton DW, Carrol AJ, et al. A common progenitor for human myeloid and lymphoid cells. Nature 274: 590, 1978.

2) Okuda T, Yokota S, Maekawa T, et al. Cytogenetic evidence for a clonal involvement of granulocyte-macrophage and erythroid lineages in a patient with refractory anaemia. Acta Haematol 80: 110, 1988.

3) Janssen JWG, Buschele M, Layton M, et al. Clonal analysis of myelodysplastic syndromes: Evidence of multipotent stem cell origin. Blood 73: 248, 1989.

4) Fialkow PJ. Clonal development and stem cell origin of leukemias and related disorders. in: Leukemia. Henderson ES, Lister TA (Eds), WB Saunders Company, Philadelphia 35, 1990.

5) Bennett J, Catovsky D, Flandrin G, et al. FAB cooperative group: Proposal for the classification of the myelodysplastic syndromes. Br J Haematol 51: 189, 1982.

6) Dameshek W. Some speculation on the myeloproliferative syndromes. Blood 6: 372, 1951.

7) Homma T, Suzuki K, Kudo Y, et al. Preparation and characterization of monoclonal antibodies against human myeloperoxidase. Arch Biochem Biophys 278: 189, 1989.

8) Beris P. Primary clonal myelodysplastic syndromes. Semin Hematol 26: 216, 1989.

9) Economopoulos T, Papageorgiou E, Hadjioannou. Refractory anaemia with excess of blasts terminating as $\mathrm{Ph}^{1}$ negative chronic myeloid leukaemia. Scand J Haematol 32: 493, 1984.

10) Yamaguchi $K$, Arimori $S$, Nagao $T$. Transformation of refractory anemia with excess of blasts into acute myelogenous leukemia with $\mathrm{Ph}$-negative chronic myelogenous leukemia-like characteristics. Am J Hematol 33: 69, 1990.

11) Ohyashiki K, Yokoyama K, Kimura Y, et al. Myelodysplastic syndrome evolving into a myeloproliferative disorder: One disease or two? Leukemia 7: 338, 1993.

12) Grigg AP, Gasciyne RD, Phillips GL, et al. Clinical, haematological and cytogenetic features in 24 patients with structural rearrangement of the $Q$ arm of chromosome 3. Br J Haematol 83: 158, 1993.

13) Bernstein R. Cytogenetics of chronic myelogenous leukemia. Semin Hematol 25: 20, 1988. 